\title{
Publisher's Note: Emittance compensation in a superconducting rf gun with a magnetic mode
} [Phys. Rev. ST Accel. Beams 7, 090702 (2004)]

K. Flöttmann, D. Janssen, and V. Volkov

(Received 23 September 2004; published 28 September 2004)

DOI: 10.1103/PhysRevSTAB.7.099901

PACS numbers: 41.60.Cr, 41.75.Fr, 42.55.Xi, 99.10.Fg

This article was published online on 22 September 2004 with an error in the spelling of the first author's last name. The first author should be "K. Flöttmann". The name has been corrected as of 24 September 2004. 